\title{
Detection of osteoporosis in lumbar spine [L1-L4] trabecular bone: a review article
}

\author{
Kavita Avinash Patil ${ }^{1 *}$, K. V. Mahendra Prashanth¹, A Ramalingaiah ${ }^{2}$
}

\begin{abstract}
${ }^{1}$ Department of Electronics and Communication Engineering, ${ }^{2}$ Department of Orthopaedic, SJBIT, Affiliated to VTU, Bangalore, Karnataka, India
\end{abstract}

Received: 07 May 2021

Revised: 01June 2021

Accepted: 02 June 2021

\section{*Correspondence:}

Dr. Kavita Avinash Patil,

E-mail: kavitamalagatti@gmail.com

Copyright: (c) the author(s), publisher and licensee Medip Academy. This is an open-access article distributed under the terms of the Creative Commons Attribution Non-Commercial License, which permits unrestricted non-commercial use, distribution, and reproduction in any medium, provided the original work is properly cited.

\begin{abstract}
The human bones are categorized based on elemental micro architecture and porosity. The porosity of the inner trabecular bone is high that is $40-95 \%$ and the nature of the bone is soft and spongy where as the cortical bone is harder and is less porous that is 5 to $15 \%$. Osteoporosis is a disease that normally affects women usually after their menopause. It largely causes mild bone fractures and further stages lead to the demise of an individual. This analysis is on the basis of bone mineral density (BMD) standards obtained through a variety of scientific methods experimented from different skeletal regions. The detection of osteoporosis in lumbar spine has been widely recognized as a promising way to frequent fractures. Therefore, premature analysis of osteoporosis will estimate the risk of the bone fracture which prevents life threats. This paper focuses on the advanced technology in imaging systems and fracture probability analysis of osteoporosis detection. The various segmentation techniques are explored to examine osteoporosis in particular region of the image and further significant attributes are extracted using different methods to classify normal and abnormal (osteoporotic) bones. The limitations of the reviewed papers are more in feature dimensions, lesser accuracy and expensive imaging modalities like computed tomography (CT), magnetic resonance imaging (MRI), and DEXA. To overcome these limitations it is suggested to have less feature dimensions, more accuracy and cost-effective imaging modality like X-ray. This is required to avoid bone fractures and to improve BMD with precision which further helps in the diagnosis of osteoporosis.
\end{abstract}

Keywords: BMD, DXA, Lumbar spine, Machine learning algorithms, Osteoporosis, T-score

\section{INTRODUCTION}

Osteoporosis is a bone disease which affects the structure of the bones as a person gets older. It weakens the strength and decreases the size of the bones, which leads to fracture. ${ }^{1}$ The anatomy of the spine is the most commonly affected part of the human body. It is divided into three major sections: the beginning part of the spine is cervical bones $(\mathrm{C} 1-\mathrm{C} 7)$, the middle part of the spine is thoracic bones (T1-T12) and the lower part of the spine is lumbar bones (L1-L5) as shown in Figure 1 and each individual bone is called vertebrae., ${ }^{2,3}$ The Trabecular (cancellous or spongy) bone quantity is $40-95 \%$ compared to the cortical (compact or dense) bones which lies between 5-15\%. The internal trabecular structures of different stages of normal, osteoporotic and osteoporotic compression fracture of the vertebra are as shown in Figure 2. Most of the body weight is carried by the lumbar spine. This gives flexibility for the movements and hence there is a chance of getting osteoporosis which commonly affects the lower part of the spine. The mortality rate of the lumbar spine is $0.13 \% .^{4}$ 
Many senior citizens are affected due to osteoporosis which causes pain, disability and it gradually leads to the death of the individual. According to a study in India, it was estimated that at present the average life span is 67 years, by 2025 it would have increased to 71 years and would see a considerable rise to 77 years by 2050. At present $10 \%$ of the Indian population is more than 50 years and it is expected that by the year 2050, the figure would rise up to $34 \% .{ }^{5}$ Therefore, in the coming years a good life span and larger proportion of people above the age of 50 will be accountable for the rise in the number of osteoporotic individuals.

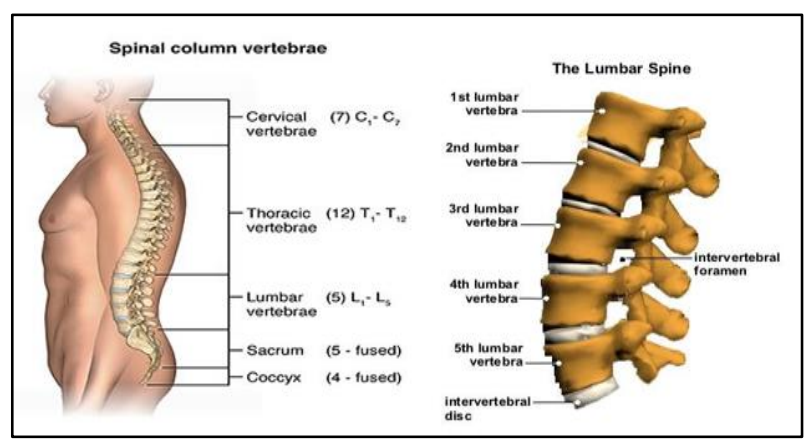

Figure 1: Anatomy of the spine. ${ }^{2,3}$

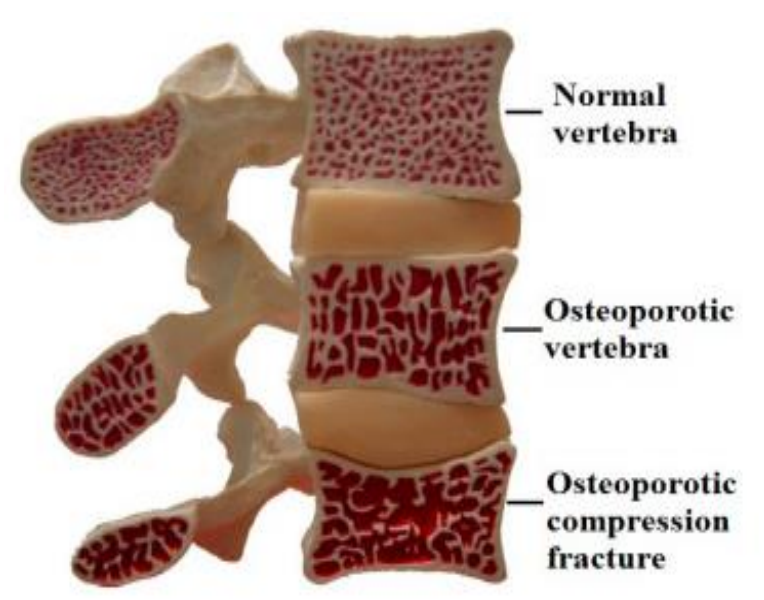

Figure 2: Stages of osteoporosis. ${ }^{48}$

Table 1: T-score of BMD. ${ }^{7}$

\begin{tabular}{|ll|}
\hline Classification & T-Score \\
\hline Normal & -1.0 or higher \\
\hline Osteopenia & Between -1.0 and -2.5 \\
\hline Osteoporosis & -2.5 or lower \\
\hline
\end{tabular}

The World Health Organization (WHO) has classified BMD measurements of T-score as shown in Table $1 .{ }^{5,7} \mathrm{~A}$ T-score measures a standard deviation of bone density variations from the average (mean), to calculate if the bone density is lower or higher than that of a healthy 30-yearold adult. According to a study conducted in 2013, it was estimated that nearly 50 million people in India had T- scores of $<-1.3 .^{6}$ The analysis concluded that less calcium consumption, deficiency in vitamin D and poor knowledge about Osteoporosis was the reason for health problems faced by majority of India women. ${ }^{5}$ The vertebral osteoporosis can be detected by comparing the test samples with the standard BMD measurements of Table 1 (based on classification of bone quality) which is a commonly exercised procedure in the clinical systems.?

\section{REGULATING FACTORS OF OSTEOPOROSIS}

Osteoporosis is quite common bone disease whose victims are older people and women who have attained menopause. Once osteoporosis affects bones, this leads to fractures which cause pain and disabilities. ${ }^{5}$ Hence it is difficult to diagnose any bone disorder of the affected individual. In postmenopausal women older than 60 years, testing of BMD measurement is based on the risk factor report, and hence the measurement of BMD is suggested. However, muscle escalation exercises and weight checking are advocated to improve the BMD. The bone regulating risk factors of osteoporosis which can be easily adaptable to avoid bone fractures are listed below. ${ }^{5}$

\section{Benefits of nutrients}

Vitamin D and calcium are the two main nutrients involved in the growth of bones. They play a vital role in maintaining the bone quality and influencing the risks due to Osteoporosis. The two nutrients are described as follows:

\section{Calcium}

It is deposited in the bone matrix, and has a crystal structure whose constituents include hydroxyl endmember of the complex apatite group crystals. Calcium is responsible for the development of strong bones and teeth. However human body cannot create calcium by its own and hence it is significant to acquire sufficient calcium from the food. When calcium is insufficient osteoporosis affects bone quality and the bone will turn pathetic and susceptible to fragility. It has been noticed that daily recommended amount $(600 \mathrm{mg} / \mathrm{d})$ of calcium is not consumed by Indian women as directed by the Medical Research Council of India. ${ }^{8}$

\section{Vitamin D}

When the human skin is exposed to sunlight, vitamin D is synthesized. Several reports suggest that Indian suffers vitamin $\mathrm{D}$ deficiency, in spite of having abundant sunlight. ${ }^{9,10}$ Deficiency of vitamin $D$ is because of insufficient exposure to sunlight, conventional clothes, insufficient dietary intake, pigmented skin and lack of intake of supplements. ${ }^{6}$ Deficiency in Vitamin D leads to low absorption of calcium from the gastrointestinal system and this affects the deposition of minerals on the bone matrix for the development of bones. 


\section{Malnutrition}

It is one of the major factors to cause osteoporosis. A woman weighing below $60 \mathrm{~kg}$ and height $155 \mathrm{~cm}$ are more prone to osteoporosis. A skeleton of suitable BMD is developed by a number of channels linking fat and bone to bear the weight. The main determining factor for BMD is the body weight and significantly the adipose tissue. Many researchers have shown a constructive relation linking BMD and body mass index. ${ }^{11}$ The major nutritional concerns in India are poor nutrition of the mother, low birth weight, highly diseased and increasing death rate in children. $^{5}$

\section{Lifestyle}

Changes in our lifestyle has also been a major factor for the loss in bone health. For example, passive living, low sun exposure and lesser physical activity. ${ }^{6}$ Muscle and bone strength can be maintained by physical exercises, prominently weight bearing exercise. Absence of exercise in the daily routine of Indian women accounts to lower BMD. ${ }^{11}$ Though smoking is considered as an important factor to cause osteoporosis yet it is observed that cigarette smoking is not so prevalent among women in India to be accountable to increase the number of osteoporotic patients.

Table 2: Osteoporosis prevalence $(\%)$ in countries. ${ }^{12}$

\begin{tabular}{|llllllllll|}
\hline $\begin{array}{l}\text { Age (in } \\
\text { years) }\end{array}$ & $\begin{array}{l}\text { India } \\
\text { Spine } \\
(\boldsymbol{\%})\end{array}$ & $\begin{array}{l}\text { Femoral } \\
\text { neck }(\boldsymbol{\%})\end{array}$ & $\begin{array}{l}\text { Korea } \\
\text { Spine } \\
(\boldsymbol{\%})\end{array}$ & $\begin{array}{l}\text { Femoral } \\
\text { neck }(\boldsymbol{\%})\end{array}$ & $\begin{array}{l}\text { Australia } \\
\text { Spine }\end{array}$ & $\begin{array}{l}\text { Femoral } \\
\text { neck }(\boldsymbol{\%})\end{array}$ & $\begin{array}{l}\text { Kuwait } \\
\text { Spine }\end{array}$ & $\begin{array}{l}\text { Femoral } \\
\text { neck (\%) }\end{array}$ \\
\hline $\mathbf{5 0 - 5 9}$ & 29 & 9.8 & 13.2 & 5.3 & 6.3 & 3.9 & 16 & 7 \\
\hline $\mathbf{6 0 - 6 9}$ & 45 & 27.2 & 30 & 16.8 & 18 & 12.9 & 35 & 13 \\
\hline $\mathbf{7 0 - 7 9}$ & 52 & 43 & 49 & 43.4 & 31 & 28.8 & 56 & 16 \\
\hline $\mathbf{8 0}$ & 71 & 71 & 60 & 74 & 36.5 & 48.8 & 70 & 18 \\
\hline
\end{tabular}

Table 3: General age stratified prevalence of osteoporosis. ${ }^{12}$

\begin{tabular}{|lll|}
\hline Age intervals (in years) & $\begin{array}{l}\text { Femoral neck } \\
\text { (Percentage within age interval) }\end{array}$ & $\begin{array}{l}\text { Lumbar spine } \\
\text { (Percentage within age interval) }\end{array}$ \\
\hline $\mathbf{5 0 - 5 5}$ & 5.1 & 26.3 \\
\hline $\mathbf{5 6 - 6 0}$ & 19.8 & 39.1 \\
\hline $\mathbf{6 1 - 6 5}$ & 25.1 & 45.1 \\
\hline $\mathbf{6 6 - 7 0}$ & 43.8 & 51.9 \\
\hline $\mathbf{7 7 5}$ & 56 & 56 \\
\hline
\end{tabular}

\section{OSTEOPOROSIS IN DIFFERENT COUNTRIES}

A comparison of osteoporotic occurrence in spine and femoral neck region of a postmenopausal woman in India with respect to three other countries is as mentioned in Table 2 and it was observed that the osteoporotic individuals among Indian population (excluding rural population) is increasing with age in comparison with other countries. ${ }^{12}$ The occurrence of Osteoporosis among individuals of different age groups is as shown in Table 3 , and observed that Lumbar spine is the most affected part of the human system when compared to femoral neck.

The risk factors of Osteoporosis cohort is as shown in Table 4 and can be observed that after several studies $12.7 \%$ had suffered from fracture in the past, $10.9 \%$ were smokers, $9.5 \%$ took some form of alcohol at least once in a week, $80.3 \%$ had inactive (urban) lifestyle, $75.7 \%$ never took any nutrient (calcium or Vitamin D) supplements and $4.9 \%$ were on steroids. ${ }^{12}$ Table 5 represents the relation between BMI and percentage of people affected by Osteoporosis. Table 5 represents the relation between BMI and percentage of people affected by osteoporosis. ${ }^{12}$

\section{CHANNEL TO DETECT OSTEOPOROSIS}

The advancement in imaging systems to diagnose osteoporosis and to detect the probable fracture analysis are generally carried out in five different stages: acquire an image, pre-processing, select the region of interested (ROI) sub-image, significant feature extraction, classify the bone image according to the BMD values. In clinics, imaging techniques are used to scan images in the first stage. ${ }^{13}$ The modification of the source (raw) data being provided to the ML (machine learning) or DL (deep learning) algorithms is referred as Pre-processing. In other words, it is a technique which assists in suppressing unwanted distortions due to image scanner and enhances significant image features of the images obtained in the first stage. In the third stage, the ROI of the bone image is segmented depending on the requirements using manual segmentations, semi-automation using certain techniques, or completely automated using sophisticated but with more convenient techniques. In the fourth stage, various feature extraction techniques are introduced to extract significant features from the segmented image of ROI of trabecular and cortical bone characteristics and these features are utilized to diagnose the probability of fracture in an osteoporotic individual. Lastly (fifth stage), the bone 
is classified either on the basis of Z- score and T- score which are determined from standard values or by utilizing supervised ML algorithms for categorizing the bones as osteoporotic or healthy.

\section{DIAGNOSIS OF OSTEOPOROSIS USING QUANTITATIVE IMAGING TECHNIQUES}

The procedures used to determine bone quality of the human body and risk factors due to osteoporosis are: the magnetic resonance imaging (MRI) [14], dual energy Xray absorptiometry (DXA or DEXA), quantitative ultrasound (QUS), digital X-ray radiogrammetric (DXR), quantitative computed tomography (QCT), highresolution peripheral quantitative computed tomography (HR-pQCT) and radiography. ${ }^{13}$

\section{Table 4: Distribution of body weights of the participants. ${ }^{12}$}

\begin{tabular}{|l|l|}
\hline Risk factor & Percentage \\
\hline History of fracture & 12.7 \\
\hline Regular smokers & 10.9 \\
\hline Alcohol intake & 9.5 \\
\hline Sedentary lifestyle & 80.3 \\
\hline $\begin{array}{l}\text { Never took Vitamin D or calcium } \\
\text { supplements }\end{array}$ & 75.7 \\
\hline Steroids or anticoagulants intake & 4.9 \\
\hline
\end{tabular}

\section{MRI of the spine}

The volumetric analysis of the cancellous (trabecular) bone can be obtained using MRI which is a nonionizing technique. It benefits from the diffusive water in the porous structure of the cancellous (spongy/trabecular) bone architecture. Segmentation of trabecular bone region is obtained from MRI images and characteristic features are analyzed. ${ }^{13}$ Though MRI is favourable for the diagnoses of osteoporotic individuals yet it is not used regularly in medical field. Constraints of using MRI in clinical practices are time-consuming process, motion distortions and vulnerability towards incomplete effects of volume which results in amplifying the size of spongy bone network and in turn affects the measurements. Highresolution (HR) micro-MRI is examined for the analysis of bone structure (trabecular). It can be used for imaging at 1.5 or 3.0 Tesla. The Constraints of imaging at 1.5 Tesla is low signal to noise (SNR) and low spatial resolution. Features of trabecular bone obtained using HR-MRI is extremely correlated to pQCT (peripheral quantitative computed tomography). The quantitative magnetic resonance (QMR) can be used to assess the cortical and trabecular bone strength by measuring the properties of neighbouring bone marrow and proton MR spectroscopy (MRS) is used for analyzing the weight (fat) of the bone marrow. The MR scan provides details of the fat and water content and various compartments of fats present in the bone marrow. The dynamic contrast-enhanced MRI measures the bone marrow perfusion indices and is used to assess the bone quality as a biomarker. There is a converse relation which occurs between BMD and bone marrow fat, and a direct proportionality between BMD and bone marrow perfusion indices. ${ }^{15}$

\section{DEXA of the spine}

Bone density or strength is determined by bone density testing which is a medical procedure. This procedure can pinpoint Osteopenia (initial stage of losing bone due to calcium loss) or Osteoporosis (a stage where bone looses strength tends to less dense and brittle). DEXA system is one of the main procedures used in the medical field to determine bone density, a faster and a pain-free method. ${ }^{15}$ It utilizes a unique $\mathrm{X}$-ray scanning machine to calculate bone density with precise output (DEXA image). BMC (Bone Mineral content) is obtained from automatically segmented region of interest of the DEXA image is derived from the ratio of Bone Mineral Content to total area of region of interest and calculated in terms of $\mathrm{g} / \mathrm{cm} 3$. Hence referred to as BMD testing machine. Most doctors prescribe DXA to analyze osteoporosis and the risk of bone fracture. The DXA machine works on the basis of a number of X-rays used to determine tissue density and bone quality. The DXA transforms bone density detail into $\mathrm{T}$ score and $\mathrm{Z}$ score according to WHO standards. The $\mathrm{T}$ score determines the amount of bone density of patients in comparison to that of a healthy individual and further utilizes it to estimate the risk of bone fracture development. $\mathrm{Z}$ score is the determination of statistical variation of bone mass density in comparison with average bone mass density. These core values are able to indicate the doctor, whether the patient requires further medical tests or not. ${ }^{16}$ Distinct values of BMD can be obtained from various skeletal sites using central DEXA (lumbar spine and hip) and peripheral DEXA (forearm). Advantages of using DEXA are accurate results, low scanning period, short radiation exposure and estimates up to $70 \%$ of the bone solidity(strength). ${ }^{13}$ Constraints of DEXA are restricted availability, expensive, susceptibility towards changes in bone measurements(size).

\section{$Q C T$}

Quantitative (Volumetric) analysis of cancellous (trabecular) bone density can be determined using QCT technique. Different angles at which the $\mathrm{X}$-rays are attenuated by the tissues over the Region of interest are captured in QCT. To convert the attenuated measurements (Hounsfield units) into BMD values (mg hydroxyapatite/cc) a model (phantom) of distinct densities of bone is positioned close to the Osteoporotic individual throughout the acquisition. Segmented ROI is obtained from the image, further a 3D replica is redeveloped using those segments. ${ }^{13}$ Attenuation coefficient is different for trabecular and cortical bones and hence can be distinguished easily. Cancellous Bone density (at hip and spine) can be determined using QCT. Calibration standard is utilized to obtain precise output over Computed Tomography scanners from various manufacturers. CT displays accurate quantitative (volumetric) measurements 
of Bone Mineral Density free of body parameters (size). Calculation of cancellous (trabecular) bone density using QCT is comparatively more susceptible than that of DEXA Bone Mineral Density. Osteoporotic (degenerative diseases) and obese patients can be diagnosed using QCT. The predicted hip fractures at the femur using QCTtrabecular BMD of the aged is equivalent to that of DEXA -BMD. QCT is expensive and has a higher radiation exposure. Through the acquisition of image, artifacts can fiddle with the image there by decreasing precision. The WHO standards of Classification of Osteoporosis cannot be obtained for the T-scores measured using QCT as it is lesser than DEXA.

\section{Table 5: Distribution of body weights of the participants. $^{16}$}

\begin{tabular}{|ll|}
\hline Weight/BMI (body mass index) & Percentage \\
\hline Underweight (BMI is $\mathbf{1 8 . 5})$ & 7.7 \\
\hline Normal weight (BMI is 18.5-24.9) & 59.6 \\
\hline Flabby weight (BMI is 25-29.9) & 23 \\
\hline Fat (BMI is 30 or more) & 9.6 \\
\hline
\end{tabular}

The technique where the QCT scanned image of the proximal femur is estimated as a two-dimensional (2-D) image to obtain DEXA equivalent is referred to as CTXA (Computed Tomography X Ray Absorptiometry). The values of areal CTXA are utilized for classification (on the basis of WHO standards) and for determination of fracture risk assessment tool (FRAX) score. The precision of calculation of BMD values can be increased with the help of Dual-energy QCT in comparison with Single-Energy QCT. ${ }^{17}$ Trabecular and cortical (compact) bone micro structures can be obtained using Micro-CT at an extremely high resolution $(1-100 \mu \mathrm{m} 3)$ and which is correlated with bone characteristic (histomorphometric) determination. Higher dimensional (spatial) resolution is offered by MDCT (Multidetector CT) in comparison with spiral Computed Tomography scanner. The distal tibia attributes from MDCT are highly related to that of $\mathrm{mCT}$ (micro-CT) obtained features. Although precise calculation of bone density is determined by MDCT yet this dimensional (spatial) resolution is insufficient to image- architecture of trabecular bone. High radiation dose $(3 \mathrm{mSv})$ is required by MDCT (high-resolution). Fractured and healthy vertebral groups can be distinguished using microstructure and density attributes extracted with the help of Multidetector CT. ${ }^{15}$

\section{$H R-p Q C T$}

High resolution quantitative (volumetric) images of outlying (peripheral) body parts such as tibia and distal radius can be obtained by HR-pQCT which is a QCT technique. In the process of detecting the boundary of the bone region, the region of interest is segmented semi automatically using Edge Detection method. Thresholding method and Gaussian smoothing filter is used to obtain segmentations of cortical bone compartments. To obtain trabecular bone, cortical bone is removed from entire region of the bone. Bone architecture and bone density of cortical and trabecular bone can be measured simultaneously using HR-pQCT. Volumetric BMD is measured using calibration model same as QCT. Anisotropy and connectivity of the bone structure (architecture) is analyzed using various morphometric methods.FEA (Finite element analysis) can be carried out on the image of HR-pQCT and biomechanics of trabecular bone may be determined. Advantages of using HR-pQCT are SNR (signal-to-noise), low radiation dose compared to MDCT, high resolution images which gives minute details of architecture of trabecular and cortical bone, provides measurements of density having high duplicability with variation coefficient up to $1 \%$. Disadvantages of using pQCT is time consuming scanning period. This is because the necessity of follow up scans with higher accuracy order $(100 \mu \mathrm{m})$ leads to increasing duration of image acquisition and also distortion in the image due to slight movements of the arm or leg. The pQCT can measure 3D structural parameters which is well correlated with micro-CT having goodness of fit $(\mathrm{R} 2>0.9)$. Peripheral measurement is convenient for individuals suffering from hip or spinal fracture as well as for morbid patients. The pQCT can predict osteoporosis based on density of bone and micro structural measurements independent of DEXA. HRpQCT has capability of measuring the deviation in bone micro structure of individuals with similar values of BMD. ${ }^{13,18,19}$

\section{$Q U S$}

It is a cost-efficient, simple and radiation-free process for quantification of trabecular bone architecture and it uses a number of pulsating resonance effect to disseminate through the trabecular bone with frequency ranges from $500 \mathrm{kHz}$ to $1.25 \mathrm{MHz}$ Properties of bones are measured based on attenuation of sound waves and differential reflections. The characterization of bone properties is based on measurements such as Speed of sound (SOS) and Broadband Ultrasound Attenuation (BUA). With osteoporosis SOS decreases and value of BUA increases. Various measurements obtained by QUS like apparent integrated backscatter, stiffness index, QUS index, amplitude-dependent SOS, is useful for analyzing the risk of fracture in patients. Exclusive QUS scanners that are readily available are phalanx, tibia, and calcaneum. Among which the QUS scanners for calcaneum are widely used for texture analysis with accurate results. Survey on QUS has revealed its capability of distinguishing osteoporotic individual from healthy individuals. QUS scanners for Calcaneum are capable of predicting hip and osteoporotic fracture in aged people, free of DEXA Bone Mineral Density measurements. Advantages of QUS are measurement at the peripheral limb, no radiation, portability of equipment, low cost etc. Measurements differ with QUS of various manufacturers and with distinct skeletal sites. QUS for calcaneum is poorly associated with DEXA of spine and hip. The system is specifically pre-set with thresholds values, by correcting the adjustments for fine-tuning QUS with respect to DXA. ${ }^{20}$ Constraints of using QUS are lack of susceptibility, temperature sensitive, poor reliability, low precision and reproducible as a result of inappropriate position of transducers. ${ }^{21}$ 
Hence, confines its usage as a tool for pre-screening rather than a tool to diagnose osteoporotic patients in medical field.

\section{Digital X-ray radiogrammetry}

The combination of texture analysis technique and computerized radiogrammetry constitutes digital X-ray radiogrammetry (DXR) which can be used to determine BMD. ${ }^{22}$ It was established as a cost-efficient substitute of DEXA. Estimation of BMD of the middle three metacarpal bones by DXR utilizes one arm radiograph. Active shape model segmentation technique is utilized to obtain segmented ROI of the arm image automatically. Bone volume per area is determined using a combination of cortical porosity index (textural analysis) and radiogrammetric MCI (measurement of the cortical bone), which is further multiplied by a constant for the purpose of approximating it to DEXA BMD of forearm. Although BMD of DXR is well correlated to that of DEXA of various bone sites (hip, spine and forearm) yet it is not an accurate quantitative bone density measurement. Texture analysis of cancellous bone architecture cannot be obtained by DXR which could have yielded a more susceptible analysis.

\section{Radiography}

It is a digital x-ray used for obtaining images of bones within the body, which includes the ankle, foot, leg, knee, hip, thigh, pelvis, spine, elbow, shoulder, arm and wrist. ${ }^{14}$ It helps in the analysis of bone fracture as an effect of osteoporosis. Although various volumetric imaging techniques such as QCT, HR-pQCT, MRI, DEXA etc developed for analyzing Osteoporosis are accurate and favourable, yet these techniques are expensive and less accessible in emerging countries. Hence this limits its usage for mass screening of osteoporosis and for further assessment during medicinal treatment. These limitations are circumvented using radiography which has obtained significance due to its ease of acquisition, accessibility and affordability. Machine learning algorithms and advanced image processing techniques are employed for examining the risk of bone fracture and osteoporosis, utilizing radiographs with the development of highly processed computers. The weight bearing bones like lumbar spine, hip, wrist, dental and calcaneum (heel) radiographic images are regularly used. Segmentations of bone ROI are obtained automatically or manually. Features like texture analysis for details on connectivity, anisotropy, radiogrammetry etc are extracted from radiographs. Classifier models are trained using supervised learning techniques for distinguishing osteoporotic individuals from healthier individuals. ${ }^{13}$

\section{OSTEOPOROSIS DETECTION METHODOLOGY}

AND

Several research works have been carried out to diagnose osteoporosis from past few decades. Survey papers involving various methodologies to detect osteoporosis, published between 2010 and 2020 in English, were spotted in Med know, Medline, Wiley, Elsevier and Springer. Journal papers and reports were investigated using citation database SCOPUS and scanned manually for detection of osteoporosis. Data were extracted from the studies that fulfilled the following criteria: title and abstract of the given study that had included Osteoporosis detection, Segmentation method, Analysis of bone quality, feature extraction and various machine learning classifiers considered in the study.

Omiotek et al stated to detect osteoporosis of thoracolumbar portion of the spine using computed tomography (CT) images is carried out based on the fractal analysis to extract texture features. ${ }^{23}$ This produces a set of feature descriptors based on linear regression and three descriptors are calculated. The variation method is used to calculate two fractal dimensions from gray images and the boxcounting is used to determine a third fractal lacunarity from binary images. These feature descriptors are used to classify the osteoporotic bone (abnormal) and normal bone in CT images with the help of six different classifiers. The performance results are varied depending on the classifiers, but $\mathrm{k}-\mathrm{NN}$ ( $\mathrm{k}$ the nearest neighbors with $\mathrm{k}=10$ ) classifier gives better performance than other classifiers.

Machine learning tools (Tensor flow and Python programming) was utilized to detect osteoporosis of spine in terms of HU (Hounsfield units) of lumbar CT and information of QCT (Quantitative Computed Tomography). Several regression algorithms were used to detect osteoporotic or non-osteoporotic vertebra by calculating the T-score by independent inputs (sex, age, and Hounsfield units of vertebrae on CT) incorporated with that of QCT. Nam et al proposed that substantial increase in data set would provide more accurate results. ${ }^{24}$ The experimentally calculated recall was $96.9 \%$, which is a positive outcome predicted appropriately to all inspections of the given subject and F1-score of $95.4 \%$ is the subjective normal of Precision with Recall being better system performance. Hence, machine learning is one of the most significant modality for research in medical field.

In some cases, lumbar spine fragility detection in patients is not suitable using Dual Energy X-ray Absorptiometry (DXA) of the anteroposterior (AP) view as it is not capable of distinguishing the trabecular and cortical bone structure density. Picazo et al proposed a method which provided density estimation and 3D subject specific model of the lumbar spine using a single anteroposterior (AP) DEXA image. ${ }^{25}$ A 3D density and statistical model was built utilizing training set of QCT scans which was further registered on to AP-DEXA image so that its projection matched. Trabecular and cortical bone compartments was segmented using model-based algorithm. On comparison of DEXA derived with QCT extracted 3D calculation for proof set of 180 samples. Precision in shape at vertebral body and total vertebra are $0.66 \mathrm{~mm}$ and $1.51 \mathrm{~mm}$ respectively. Coefficients of Correlation between QCT and DXA derived estimation extended from 0.81-0.97. This model improved the detection of osteoporosis and 
fracture risk assessment better than AP DXA scan without any further assessment.

Shaker et al stated that recurrent neural network was used to predict osteoporosis of AP lumbar spine using MRI. ${ }^{26}$ This network was implemented with five different models, the first three were custom models designed to characterize different depths, and the remaining two models were designed for fine-tuning at different levels. This design process increased the representational capacity to analyze osteoporosis with better fracture prediction and bored the convenience of transfer learning which included fast training process and aptness for larger datasets. There were some practical challenges for deep learning for instance lower training process and insufficiencies of precision was conveyed.

Auto-diagnostic techniques made use of morphometric features extracted from CT images to measure three parts of vertebral structure for diagnosis of vertebral compression and anomaly was located using segmentation and vertebral edges was determined. Yousefi et al recommended procedure consisted of noise reduction and preference to the best slice of CT image, identification of the lumbar region in the spine, estimation of six points on the contour of the vertebral body and each vertebral morphometric features such as crush, wedge and biconcave were extracted to be used in the diagnosis of vertebral compression fractures. ${ }^{27}$ Osteoporotic and normal bones were classified based on SVM and KNN classifiers to obtain better system performance. KNN classifier $(\mathrm{k}=5)$ is better than SVM. It can be concluded from survey paper that the challenging task for researchers is to collect datasets which improves system performance and alignment of images.

Korchiyne et al proposed Machine learning-based Legendre's Multifractal spectrum model was developed to diagnose osteoporosis in CT and MRI images. ${ }^{28}$ This system was developed to extract the texture features of the trabecular bone structure using Multifractal features and Support vector machine to diagnose osteoporosis and normal bones. Here by improving the system performance.

Wang et al suggested a method for acquiring features such as volumetric parameter (morphological) and bone density determinants to classify vertebral compression fractures (VCF) of Osteoporotic origin. ${ }^{29}$ Although it is observed from the data that misclassifications produced by longitudinal feature set is more in comparison with other features (Demographic and measured) yet on inclusion of longitudinal feature in committee of SVM, provides ease to accurate classification but advancement is not significant by data.

The T1WI - weighted MRI scanned image in the sagittal plane is used to detect the vertebral compression fractures (VCF) of the lumbar spine. The features like Fourier descriptor, HU's moments, convex deficit, and normalized compactness of shape features are extracted then a combination of the different features is used to classify the $\mathrm{VCF}$ with the help of $\mathrm{KNN}(\mathrm{k}=3)$ classification techniques. Frighetto-Pereira et al advised model gives better shape analysis to discriminate between fractured and normal vertebral bodies. ${ }^{30}$ Various texture and shape features with semi-automated segmentation of lumbar vertebrae must enhance the complete processing chain leading to better classification in diagnosis of VCFs benign versus malignant.

The hybrid type classifier model is developed to diagnose osteoporosis using an artificial neural network (ANN) based monarch butterfly optimization (MBO) technique built on individual attribute values like trabecular separation, trabecular number, age, body mass index, etc. Devikanniga et al suggested model is experimented and verified by ten-fold cross-validation of lumbar spine data sets. ${ }^{31}$ Outcomes of the model are better analogized using receiver operating characteristics (ROC) and Wilcoxon signed-rank verified with existing approaches.

Paper by Valentinitsch et al is based on combination of three-dimensional texture features extracted using wavelets (WL), local binary patterns (LBP), histogram of gradients (HoG), gray-level co-occurrence matrix Haralick features (HAR) and local volumetric BMD of CT images to detect osteoporosis. ${ }^{32}$ These features are registered on to random forest classifier which is a high discriminative applied to improve the overall system performance. The fourfold cross-validation is conducted for system verification to distinguish the features for significant improvements.

Krishnaraj et al describes that in comparison with DEXA, usage of CT is high and it provides useful datasets for diagnosis of Osteoporosis. ${ }^{33}$ The motive is to obtain accurate Dexa scores of Lumbar regions using CT based on ML algorithms. The regression-based model is developed to resolve the correlation between DEXA Tscore and grade calculation for L1-L4. Tang et al proposed method includes automatic detection of osteoporosis in the lumbar vertebra using CT image based on the convolutional neural network $(\mathrm{CNN})$ consisting of two methodologies namely 1) mark segmentation network (MS Net) and 2) bone conditions classification network (BCC Net). ${ }^{34}$ The MS net is trained to locate and mark the specified lumbar vertebra for an input $\mathrm{CT}$ image and then slice the region of interest (ROI). The BCC-Net is trained to utilize the extracted features of ROI to classify bone conditions and then it calculates the probability of Osteopenia, Osteoporosis and normal bone mass by inserting the sliced image. The network achieves very good results and performs better segmentation in terms of shapes.

Lee et al explored different machine learning models to forecast BMD utilizing DEXA and X-ray image features of spine obtained by three deep learning algorithms. ${ }^{35}$ Systems to predict highly risked society with abnormal 
BMD was identified. This system achieves 0.75 Recall and $0.73 \mathrm{~F} 1$-score for its better performance.

Saad et al performed a quantitative study on detection of lumbar spine osteoporosis using MRI-based score (Mscore) and calculation of relative correlation between lumbar spine signal intensity measured from MRI on sagittal plane of T1WI, T2WI and BMD, Z-score, T-score from DEXA in post-menopausal women. The statistical quantitative method significantly improves distinguishing osteoporotic from non-osteoporotic bones. ${ }^{36}$

Muehlematter et al utilized texture analysis attributes of vertebral bone from $\mathrm{CT}$ images to detect risk of bone fracture using open-source software (MaZda). ${ }^{37}$ The study cases and the matched controls were classified utilizing ROC portion of the image texture features analyzed using Hounsfield unit (HU) and supervised ML techniques. A combination of texture analysis along with ML in CT enhances precise identification of vertebral fractures.

Zhao et al investigated prognostic values of utilizing BMFF (bone marrow fat fraction) of vertebrae obtained from mDixon (modified Dixon) quant to determine abnormal and osteoporotic bone density. ${ }^{38}$ The mDixon Quant is a nonionizing, non-invasive, fast and a simple process to examine BMFF of vertebrae. High predictive power of mDixon Quant facilitates in the detection of Osteoporosis and abnormal bone density. A moderate inverse co-relation was found between BMFF and BMD.

Frighetto - Pereira et al analyzed vertebral compression fractures (VCFs) in T1-weighted MRI images to identify insufficient bone density due to osteoporosis which leads to lumbar vertebral metastasis i.e., malignant fractures. ${ }^{39}$ Initially lumbar vertebrae are segmented manually and gray level features are obtained statistically using histogram. Contours of vertebrae are also analyzed on the basis of texture and shape features. The KNN, neural network with radial basis function and naive Bayes classifiers are applied to classify the osteoporosis and normal bone features. This model achieves better ROC and good discrimination between osteoporosis and normal bone.

Kilic et al used twenty-four different human attributes like age, BMD, bone area, Z-score, T-score, etc to classify normal, Osteopenia and osteoporotic bones. Bagging, gradient boosting (GB) and random subspace (RSM) techniques are used to obtain features. Random forest (RF) and instance-based learning (IBL) ensemble are used in the classification of bones (healthy, osteoporosis, and Osteopenia bone). ${ }^{40}$ This ensemble learning technique is effective in the discrimination of various features (pattern) of bone.

Sungkhun et al utilized x-ray images to categorize diseases such as Osteopenia and osteoporosis of the spine by five major steps. ${ }^{41}$ Automatic Cropping of the spine image in the $\mathrm{x}$-Ray and locating lumbar spine using segmentation with the help of normal distribution and vertical projection. Distance regularized intensity position progress and Gamma corrections (GC) are used for contour identification. Estimating vertebral bones of different poses using a couple of graph peaks. Locating the lumbar spine using linear equation (LE). Identifying bone Lesion using average intensities. The overall accuracy is suitable to identify bone Lesion. Aventaggiato et al aimed to obtain an explicit validation of a completely automated algorithm for segmentation of vertebral interface in echographic images. ${ }^{42}$ Abdominal echographic scanning of lumbar vertebrae (L1-L4) of 150 women of various age and BMI were determined. The obtained data was automatically computed by the algorithm and precision of segmentations was calculated by three experienced systems. The outcome of this method had very good specific detection combined with reasonable susceptibility.

Casciaro et al proposed evaluating the efficiency of ultrasound (US) parameters in the determination of BMD of lumbar spine when used immensely in clinical field to examine the aged of various BMI ${ }^{43}$ Samples of abdominal Echographic scan and DXA images with relevant unfiltered radio frequency information are used for diagnosis of Osteoporosis. A sequence of spectral with statistical investigation is used to compute analytical parameters to determine the osteoporosis score (OS). An outstanding and an efficient relationship was established among DXA values and OS determined BMD to classify bones (healthy, Osteopenia, and osteoporosis).

Tay et al utilized the relation of Hounsfield units (HFU) to bone density and determined aBMD from CT volume. ${ }^{44}$ The obtained aBMDCT is correlated with that of DEXA derived $\mathrm{aBMD}$ and further registered for the analysis of Osteopenia of Lumbar vertebrae using statistical processes. aBMD DXA and aBMDCT is highly correlated with r-factor $=0.852$, and with RMSE (root mean square error) of $0.0884 \mathrm{~g} / \mathrm{cm} 2$. This method provides a high correlation of cortical to trabecular bone components and even in every segment in the dCT volume thus helping in the classification of osteoporotic bone. Classifier computes with precision of $80.1 \%$ and AUC of 0.894 .

Al-Helo et al proposed a completely automated CAD (Computer-Aided Diagnosis) design for the analysis of VWCF (vertebra wedge compression fracture) from CT images that incorporates within the clinical systems. ${ }^{45}$ In this method vertebra is located, labeled, segmented and diagnosed. A coordinated system including Active shape (AS) model and a gradient vector flow active contours (GVF-Snake) model is utilized for labelling and segmentation of vertebrae. This method is a clinically motivated set of features based on two machine learning solutions such as unsupervised K-Means and neural networks (NN) used to distinguish fractured vertebrae. This system has been validated with a set of twenty normal and thirty abnormal CT images with accuracy over $90 \%$. 
Pickhardt et al examined the usefulness of lumbar spine reduction amount for evaluation of BMD at CTC (Computed tomographic Colonography) scanning with DXA as a standard reference. ${ }^{46}$ The assessment of both phantoms QCT and ROI reduction amount of the lumbar spine (T12-L5 levels) are efficient for screening BMD at CTC with high sensitivity for osteoporosis with standard DXA T-score.

Saville introduced Saville Index which is used in the prediction of osteoporosis by analyzing of trabecular micro architecture patterns in radiographic images of spine. ${ }^{47}$ It examines stretch striations and endplates of vertebrae and then it classifies into five different categories depending on Saville Index to detect osteoporosis. Manual assessment of radiographs resulted in high subjective error. Hence Saville Index of trabecular micro architecture pattern indices are rarely used in medical field.

\section{DISCUSSION}

Most of the research work is carried out for the analysis of osteoporosis to detect bone fractures in early stages. Trabecular bone (spongy bone) is affected prior to hard cortical bones. Comparison of system performance of various methods used in the recent years is mentioned in Table 6. The letter $\mathrm{N}$ in the table is used to denote the data that is not available in previous research papers. Depending on the methods adapted, imaging modalities, classifiers, cross validation, accuracy, sensitivity and specificity of the system varies. Detection of osteoporosis using various Imaging modalities plays a very important role to improve the system performance. Among all classifiers mentioned in Table 6 , the hybrid, IBL and RF method of classification techniques give better system accuracy.

Table 6: Performance comparison with different methods.

\begin{tabular}{|c|c|c|c|c|c|c|}
\hline Methods and features & $\begin{array}{l}\text { Imaging } \\
\text { modalities }\end{array}$ & Classifiers & Accuracy & Sensitivity & Specificity & $\begin{array}{l}\text { Cross } \\
\text { validation }\end{array}$ \\
\hline Fractal analysis $^{23}$ & CT & k-NN & 81 & 78 & 90 & 3 -fold \\
\hline Morphometric $^{27}$ & $\mathrm{CT}$ & k-NN & 88.3 & 92.5 & 83.3 & 10-fold \\
\hline Multifractal $^{28}$ & $\mathrm{CT}$ and MRI & SVM & 95.2 & $\mathrm{~N}$ & $\mathrm{~N}$ & 10-fold \\
\hline $\begin{array}{l}\text { Morphometric with } \\
\text { global descriptors }\end{array}$ & $\mathrm{CT}$ & SVM & 82.0 & $\mathrm{~N}$ & $\mathrm{~N}$ & 10 -fold \\
\hline $\begin{array}{l}\text { Morphometric with } \\
\text { fourier descriptors }^{30}\end{array}$ & MRI & k-NN & 90.58 & $\mathrm{~N}$ & $\mathrm{~N}$ & 10-fold \\
\hline MBO-ANN approach ${ }^{31}$ & $\begin{array}{l}\text { Person attribute } \\
\text { values }\end{array}$ & Hybrid & $97.9 \pm 0.14$ & $98.33 \pm 0.03$ & $\begin{array}{l}98.33 \pm \\
0.03\end{array}$ & 10-fold \\
\hline $\begin{array}{l}\text { 3D feature with } \\
\text { vBMD }\end{array}$ & $\mathrm{CT}$ & $\begin{array}{l}\text { Random } \\
\text { forest }\end{array}$ & - & 77 & 78 & 4-fold \\
\hline $\begin{array}{l}\text { Regression based } \\
\text { model }^{33}\end{array}$ & DEXA and CT & $\begin{array}{l}\text { Random } \\
\text { forest }\end{array}$ & 82 & 84.4 & 72.7 & 2.7-fold \\
\hline $\mathrm{CNN}^{34}$ & $\mathrm{CT}$ & $\begin{array}{l}\text { BCC-net } \\
\text { MS-net }\end{array}$ & 76.65 & $\mathrm{~N}$ & $\mathrm{~N}$ & $\begin{array}{l}\text { 7- fold } \\
\text { 2- fold }\end{array}$ \\
\hline $\mathrm{DCN}^{35}$ & $\begin{array}{l}\text { DEXA and X- } \\
\text { ray }\end{array}$ & $\begin{array}{l}\text { SVMC, } \\
\text { KNN, RFC }\end{array}$ & 71 & 81 & 60 & 4-fold \\
\hline $\begin{array}{l}\text { Statistical quantitative } \\
\text { analysis }^{36}\end{array}$ & MRI and DEXA & 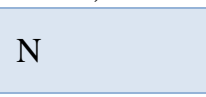 & $\mathrm{N}$ & 94 & 60 & 2-fold \\
\hline mDixon $^{38}$ & QCT & $\begin{array}{l}\text { Logistic } \\
\text { Regression }\end{array}$ & 88.7 & 87.7 & 89.6 & 3- fold \\
\hline Histogram $^{39}$ & MRI & $\begin{array}{l}\text { KNN } \\
\text { Naive Bayes } \\
\text { RBF }\end{array}$ & $\begin{array}{l}92 \\
90.6 \\
91.1\end{array}$ & $\mathrm{~N}$ & $\mathrm{~N}$ & 10 -fold \\
\hline Bagging, RS and GB ${ }^{40}$ & $\begin{array}{l}\text { Human } \\
\text { attributes }\end{array}$ & IBL, RF & 98.85 & $\mathrm{~N}$ & $\mathrm{~N}$ & 10- fold \\
\hline GC and $L^{41}$ & X-Ray & $\mathrm{N}$ & 76.25 & 50 & 65.5 & $\mathrm{~N}$ \\
\hline $\begin{array}{l}\text { Cluster with } \\
\text { thresholding }\end{array}$ & $\begin{array}{l}\text { Ultrasound } \\
\text { image }\end{array}$ & $\begin{array}{l}\text { Expert } \\
\text { operator }\end{array}$ & 78.3 & 68.1 & 93.3 & $\mathrm{~N}$ \\
\hline $\begin{array}{l}\text { Spectral, Statistical } \\
\text { search }^{43}\end{array}$ & $\begin{array}{l}\text { Echographic } \\
\text { scan and DXA }\end{array}$ & Probability & 90.1 & $\mathrm{~N}$ & $\mathrm{~N}$ & $\mathrm{~N}$ \\
\hline $\begin{array}{l}\text { HFU, statistical } \\
\text { process }^{44}\end{array}$ & DXA and CT & aBMD & 80.1 & 73.9 & 17.1 & $\mathrm{~N}$ \\
\hline AS and GVF-snake ${ }^{45}$ & $\mathrm{CT}$ & $\mathrm{NN}$ & 93.2 & 87.5 & 99.1 & 5- fold \\
\hline
\end{tabular}


Table 7: Comparison of AUC for Different methods.

\begin{tabular}{|c|c|c|c|c|}
\hline Methods & Image type & Accuracy & $\begin{array}{l}\text { Area under } \\
\text { the curve } \\
\text { (AUC) }\end{array}$ & Cross validation \\
\hline $\begin{array}{l}\text { Multivariable regression } \\
\text { algorithm }^{24}\end{array}$ & $\mathrm{CT}$ and QCT & 92.5 & 90 & 4-fold \\
\hline $\begin{array}{l}\text { Morphometric features with } \\
\text { Fourier descriptors }^{30}\end{array}$ & MRI & 90.58 & 93.7 & 10-fold \\
\hline $\mathrm{CNN}^{34}$ & CT & 76.65 & 91.67 & $\begin{array}{l}\text { 2-fold } \\
7 \text {-fold }\end{array}$ \\
\hline $\mathrm{DCN}^{35}$ & DEXA and X-ray & 71 & 0.74 & K-fold \\
\hline Statistical quantitative analysis ${ }^{36}$ & MRI and DEXA & $\mathrm{N}$ & 90.4 & 50 \\
\hline TA with ML (MaZda) $)^{37}$ & CT & $\mathrm{N}$ & 83 & 10 -fold \\
\hline mDixon $^{38}$ & BMFF & 88.7 & 94 & 88.7 \\
\hline Histogram $^{39}$ & MRI & $\begin{array}{l}\text { KNN } \\
\text { Naive Bayes } \\
\text { RBF }\end{array}$ & $\begin{array}{l}95 \\
97 \\
94\end{array}$ & 10 -fold \\
\hline HFU, statistical process ${ }^{44}$ & DXA, CT & 80.1 & 89.6 & 10-Fold \\
\hline
\end{tabular}

Table 8: Comparison of statistical variations for different methods.

\begin{tabular}{|c|c|c|c|c|c|c|}
\hline $\begin{array}{l}\text { Reference } \\
\text { No. }\end{array}$ & Techniques & Mean \pm Std de & viation & & & $P$ value \\
\hline \multirow{4}{*}{23} & \multirow{2}{*}{$\begin{array}{l}\text { Variation. } \\
\text { (Transect first } \\
\text { differences) }\end{array}$} & Healthy & & Osteoporotic & & \multirow{4}{*}{0.0129} \\
\hline & & $2.14 \pm 0.01$ & & $2.12 \pm 0.02$ & & \\
\hline & $\begin{array}{l}\text { Variation } \\
\text { (Transect second } \\
\text { differences) }\end{array}$ & $2.55 \pm 0.02$ & \multicolumn{3}{|c|}{$2.51 \pm 0.04$} & \\
\hline & Box counting & $0.27 \pm 0.02$ & & $0.29 \pm 0.02$ & & \\
\hline \multirow{5}{*}{38} & \multirow{5}{*}{ mDixon } & & $\begin{array}{l}\text { Normal bone } \\
\text { density }\end{array}$ & Osteopenia & Osteoporotic & $P$ value \\
\hline & & $\begin{array}{l}\text { Age (years } \\
\text { old) }\end{array}$ & $\begin{array}{l}39.2 \pm 12.6 \\
(37.0-41.3)\end{array}$ & $\begin{array}{l}58.4 \pm 8.2 \\
(56.6-60.2)\end{array}$ & $\begin{array}{l}62.1 \pm 5.7 \\
(60.2-63.9)\end{array}$ & $<0.01$ \\
\hline & & BMI $\left(\mathrm{kg} / \mathrm{m}^{2}\right)$ & $\begin{array}{l}22.6 \pm 3.2 \\
(22.0-23.1)\end{array}$ & $\begin{array}{l}23.2 \pm 2.4 \\
(22.7-23.7)\end{array}$ & $\begin{array}{l}23.7 \pm 3.4 \\
(22.7-24.8)\end{array}$ & 0.062 \\
\hline & & $\begin{array}{l}\text { BMD } \\
\left(\mathrm{mg} / \mathrm{cm}^{3}\right)\end{array}$ & $\begin{array}{l}159.7 \pm 31.1 \\
(154.4-165.0)\end{array}$ & $\begin{array}{l}101.1 \pm 11.5 \\
(98.5-103.6)\end{array}$ & $\begin{array}{l}66.2 \pm 11.0 \\
(62.7-69.8)\end{array}$ & $<0.001$ \\
\hline & & $\operatorname{BMFF}(\%)$ & $\begin{array}{l}41.1 \pm 9.6 \\
(39.5-42.8)\end{array}$ & $\begin{array}{l}54.9 \pm 6.9 \\
(53.4-56.4)\end{array}$ & $\begin{array}{l}60.5 \pm 6.6 \\
(58.4-62.7)\end{array}$ & $<0.001$ \\
\hline
\end{tabular}

The accuracy is the amount of proximity of measurements of a quantity to that quantity's exact value. Sensitivity of a system represents how the system output reacts to the acceptable or its behaviour to every input response. Specificity is the likelihood estimation of classifying subjects without correct negatives. Sensitivity and specificity are important parametric measures to assess the system quality. Table 7 shows the comparison of area under curve (AUC) for different methods used by the system is the area under the receiver operating characteristics, which notifies how large amount of model is able to discriminate positive classes. Higher the AUC, higher is the improvement in model prediction i.e., the model is able to discriminate between normal and abnormal bone. AUC values also depend on the methods used to analyze osteoporosis and imaging modality.
Among mentioned methods in Table 7, MRI gives better outcomes. The $\mathrm{K}$-fold validation is defined as a validation technique which prevents over fitting of predictive model to the training-data. Some researchers use mean and standard deviation measurements to detect osteoporosis in bones as mentioned in Table 8. It consists of comparison of statistical variations for different methods but these statistical variations depend on the ROI of the image and also on the number of subjects used to detect osteoporosis.

\section{Future work}

There are still potential challenges in the research field to come out with new techniques to achieve detailed information about micro architecture of trabecular bone with cost-effective diagnostic tools required to diagnose 
osteoporosis. Looking at the opportunities to predict osteoporosis and advancement in the technologies it is possible to investigate the true measurements of volumetric bone density (BMD) and would also help the global population to reduce their disabilities. This analysis is used to obtain bone indices which classifies the bone quality and also describes new methods of extracting trabecular bone texture features to evaluate the standard methods to classify the bones. Hence, developing a system for an early diagnosis of osteoporosis in order to improve the analysis of accuracy, reliability, sensitivity, correct geometric measurements, strength evaluation and prediction of risk of bone fracture is significant. This could remarkably help medical field in advancements of clinical trials, cost management and also in accounting the number of osteoporotic fractures in health system. Hence it reduces the financial burden in the upcoming years.

\section{CONCLUSION}

Osteoporosis is a metabolic bone disease, described as a less BMD and micro-architectural weakening of bone tissue, with a resulting in bone fragility and fact of being likely to fractures. This disease makes human lead a lower quality of life, makes them ad-just to disabilities and puts the health insurance systems of the countries in financial trouble. Osteoporosis can be prevented by early detection leading to early treatment which can prove helpful to women for a better quality of life. Many technologies are currently working to diagnose osteoporosis to evaluate skeletal healthiness. Furthermore, to guarantee that the legitimate imaging methodology is utilized for proper diagnose. One of them is DEXA and it is put to more regular usage. It deals with hip, spine, and total body with faster, accurate and highly precise BMD of a particular region. Although DXA is standard method of analyzing osteoporosis. It has certain drawbacks such as low accessibility, very costly and 2-D processing techniques with in-built limitations such as it cannot differentiate between trabecular and cortical bone and the changes due to bone geometry. Hence, integration of BMD measurement with texture attribute analysis of the trabecular bone will afford a better and additional responsive evaluation for premature detection of osteoporosis. Even though MRI and QCT techniques results in a true bone mass density, but have limitations like expensive cost.

\section{ACKNOWLEDGEMENTS}

This work was supported by Dr. A Ramalingaiah M.S (ortho)Orthopedic Doctor, Bangalore. No.271 Abhilasha Orthopaedic Hospital 5th Block 100ft Rd Banashankari 3rd Stage Bangalore - 560085, Bangalore.

Funding: No funding sources Conflict of interest: None declared Ethical approval: Not required

\section{REFERENCES}

1. Peterson, Anthony J. Osteoporosis overview. Geriatric Nursing. 2001;22(1):17-23.

2. https://comportho.com/spine/anatomy-of-the-spine/. Accessed on $7^{\text {th }}$ April 2021.

3. https://sites.google.com/site/activecarephysiotherap yclinic/lumbar-spine-diseases. Accessed on $7^{\text {th }}$ April 2021.

4. Poorman, Wyatt G. Rates of mortality in lumbar spine surgery and factors associated with its occurrence over a 10-year period: a study of 803,949 patients in the Nationwide Inpatient Sample. International Journal of Spine Surgery. 2018;12(5):617-23.

5. Khadilkar, Anuradha V, Mandlik RM. Epidemiology and treatment of osteoporosis in women: an Indian perspective. International journal of women's health. 2015;7:841-50.

6. Ambrish M. The Asia-pacific regional auditepidemiology, costs, and burden of osteoporosis in India 2013: a report of international osteoporosis foundation." Indian journal of endocrinology and metabolism. 2014;18(4):449.

7. Bhaskar B, Phukan P, Sarma K. Prevalence of osteoporosis among vulnerable adults residing in the north-eastern region of India: A preliminary report from a tertiary care referral hospital. Journal of Orthopaedics, Traumatology and Rehabilitation. 2017;9(2):84.

8. Nutrient Requirements and Recommended Dietary Allowances for Indians: A Report of the Expert Group of the Indian Council of Medical Research; 2009. Hyderabad: National Institute of Nutrition; Indian Council of Medical Research.

9. Khadilkar A, Das G, Sayyad M. Low calcium intake and hypovitaminosis D in adolescent girls. Arch Dis Child. 2007;92(11):1045.

10. Khadilkar AV. Vitamin D deficiency in Indian adolescents. Indian Pediatr. 2010;47(9):755-6.

11. Aggarwal N, Raveendran A, Khandelwal N. Prevalence and related risk factors of osteoporosis in peri- and postmenopausal Indian women. J Midlife Health. 2011;2(2):81-5.

12. Binu, John A. Bone Health after fifth decade in rural ambulatory South Indian postmenopausal women. Indian journal of community medicine: official publication of Indian Association of Preventive \& Social Medicine. 2019;44(3):205.

13. Areeckal AS, M Kocher SDS. Current and Emerging Diagnostic Imaging-Based Techniques for Assessment of Osteoporosis and Fracture Risk. in IEEE Reviews in Biomedical Engineering. 2019;12:254-68.

14. https://www.radiologyinfo.org/en/info.cfm?pg=oste oporosis. Accessed on $7^{\text {th }}$ April 2021.

15. Link TM. Osteoporosis imaging: state of the art and advanced imaging. Radiology. 2019;263(1):3-17.

16. Kanis JA, McCloskey EV, Johansson H, Oden A, Melton LJ, Khaltaev N. A reference standard for the 
description of osteoporosis. Bone. 2008;42(3):46775.

17. Emami A, Ghadiri H, Rahmim A, Ay M. A novel dual energy method for enhanced quantitative computed tomography. J Instrum. 2018;3(1):P01030.

18. deJongetal JJ. Fracture repair in the distal radius in postmenopausal women: Afollowup2yearspostfractureusingHRpQCT. J. Bone Mineral Res. 2016;31(5):1114-22.

19. Kazakia GJ, Burghardt AJ, Link TM, Majumdar S. Variations in morphological and biomechanical indices at the distal radius in subjects with identical BMD. J. Biomechanics. 2011;44(2):257-66.

20. Hans D, Hartl F, Krieg M. Device-specific weighted T-score for two quantitative ultrasounds: Operational propositions for the management of osteoporosis for 65 years and older women in Switzerland. Osteoporosis Int. 2003;14(3):251-8.

21. Njeh C. Comparison of six calcaneal quantitative ultrasound devices: Precision and hip fracture discrimination. Osteoporosis Int. 2000;11(12):105162.

22. Rosholm A, Hyldstrup L, Baeksgaard L, Grunkin M, Thodberg H. Estimation of bone mineral density by digital X-ray radiogrammetry: Theoretical background and clinical testing. Osteoporosis Int. 2001;12(11):961-9.

23. Omiotek Z, Dzierżak R, Uhlig S. Fractal analysis of the computed tomography images of vertebrae on the thoraco-lumbar region in diagnosing osteoporotic bone damage. Proceedings of the Institution of Mechanical Engineers, Part H: Journal of Engineering in Medicine. 2009;233(12):1269-81.

24. Nam K, Seo I, Kim D, Lee J, Choi B, Han I. Machine Learning Model to Predict Osteoporotic Spine with Hounsfield Units on Lumbar Computed Tomography. Journal of Korean Neurosurgical Society. 2019; 62(4):442-9.

25. Picazo ML. 3-D Subject-Specific Shape and Density Estimation of the Lumbar Spine from a Single Anteroposterior DXA Image Including Assessment of Cortical and Trabecular Bone. IEEE Transactions on Medical Imaging. 2018;37(12):2651-62.

26. Shaker AS. Detection and Segmentation of Osteoporosis in Human Body using Recurrent Neural Network. IJAST. 2020;29(2);1055-66.

27. Yousefi H, Salehi E, Sheyjani OS, Ghanaatti H. Lumbar Spine Vertebral Compression Fracture Case Diagnosis Using Machine Learning Methods on CT images. 2019 4th International Conference on Pattern Recognition and Image Analysis (IPRIA), Tehran, Iran. 2019;179-84.

28. Korchiyne R. New Approach Based on Multifractal Spectrum Features for Detection of Osteoporosis. 2018 International Symposium on Advanced Electrical and Communication Technologies (ISAECT), Rabat, Morocco. 2018;1-5.

29. Wang Y, Yao J, Burns JE, Summers R. Osteoporotic and neoplastic compression fracture classification on longitudinal CT. 2016 IEEE 13th International
Symposium on Biomedical Imaging (ISBI), Prague. 2016;1181-4.

30. Frighetto-Pereira L, Menezes-Reis R, Metzner GA, Rangayyan RM, Nogueira-Barbosa MH, AzevedoMarque PM. Classification of vertebral compression fractures in magnetic resonance images using shape analysis. 2015 E-Health and Bioengineering Conference (EHB), Iasi. 2015;1-4.

31. Devikanniga, D, Raj JS. Classification of osteoporosis by artificial neural network based on monarch butterfly optimisation algorithm. Healthcare technology letters vol. 2018;5(2);70-5.

32. Valentinitsch A, Trebeschi S, Kaesmacher J. Opportunistic osteoporosis screening in multidetector CT images via local classification of textures. Osteoporos Int. 2019;30:1275-85.

33. Czum, Julianna M. Simulated Lumbar Dual-Energy X-Ray Absorptiometry With Machine Learning Algorithms: Why Radiologists Who Interpret Abdominal CTs Should Care. Journal of the American College of Radiology. 2019;16(10):14712.

34. Tang, Chao, Zhang W, Li H, Li L, Li Z, Cai A et al. CNN-based Automatic Detection of Bone Conditions via Diagnostic CT Images for Osteoporosis Screening. arXiv preprint arXiv. 2019;1910.06777.

35. Lee, Sangwoo, Choe EK, Kang HY, Yoon JW, Kim HS. The exploration of feature extraction and machine learning for predicting bone density from simple spine X-ray images in a Korean population. Skeletal Radiology. 2020;49(4):613-8.

36. Saad MM, Ahmed AT, Mohamed KE. Role of lumbar spine signal intensity measurement by MRI in the diagnosis of osteoporosis in post-menopausal women. Egypt J RadiolNucl Med. 2019;50:35.

37. Muehlematter UJ, Mannil M, Becker AS, Vokinger KN, Finkenstaedt T, Osterhoff $\mathrm{G}$ et al. Vertebral body insufficiency fractures: detection of vertebrae at risk on standard CT images using texture analysis and machine learning. EurRadiol. 2019;29(5):2207-17.

38. Zhao Y, Huang M, Ding J, Zhang X, Spuhler K, Hu $\mathrm{S}$ et al. Prediction of Abnormal Bone Density and Osteoporosis From Lumbar Spine MR Using Modified Dixon Quant in 257 Subjects With Quantitative Computed Tomography as Reference. J MagnReson Imaging. 2019;49(2):390-9.

39. Frighetto-Pereira, Lucas. Shape, texture and statistical features for classification of benign and malignant vertebral compression fractures in magnetic resonance images. Computers in biology and medicine. 2016;73:147-56.

40. Kilic N, Hosgormez E. Automatic Estimation of Osteoporotic Fracture Cases by Using Ensemble Learning Approaches. J Med Syst. 2016;40:61.

41. Sungkhun S, Keo C, Khoeun R, Chinnasarn K, Rasmequan S, Rodtook A. Automated multiple lesion identification on vertebral spine using modified average intensity. 2016 International Conference on Advanced Informatics: Concepts, 
Theory and Application (ICAICTA), George Town. 2016;1-6.

42. Aventaggiato M. Validation of an automatic segmentation method to detect vertebral interfaces in ultrasound images. in IET Science, Measurement \& Technology. 2016;10(1):18-27.

43. Casciaro S, Conversano F, Pisani P, Casciaro E, Muratore M. Ultrasound Osteoporosis Score: A novel parameter for the estimation of spine mineral density," 2015 6th European Symposium on Ultrasonic Characterization of Bone, Corfu. 2015;14.

44. Wei-Liang T. Osteoporosis screening using areal bone mineral density estimation from diagnostic CT images. Academic radiology. 2020;19(10):1273-82.

45. Al-Helo S, Alomari RS, Ghosh S. Compression fracture diagnosis in lumbar: a clinical CAD system. Int J CARS. 2013;8:461-9.
46. Pickhardt PJ, Lee LJ, del Rio AM. Simultaneous screening for osteoporosis at CT colonography: bone mineral density assessment using MDCT attenuation techniques compared with the DXA reference standard. J Bone Miner Res. 2011;26(9):2194-203.

47. Saville PD. The syndrome of spinal osteoporosis. Clinics Endocrinol. Metabolism. 1973;2(2):177-85.

48. Available at: http://neuros.net/en/osteoporosis. Accessed on 3 March 2021.

Cite this article as: Patil KA, Prashanth KVM, Ramalingaiah A. Detection of osteoporosis in lumbar spine [L1-L4] trabecular bone: a review article. Int J Res Orthop 2021;7:872-84. 